\title{
Measurement of charge collection in irradiated miniature sensors for the upgrade of ATLAS Phase-II Strip tracker
}

\author{
Vladimir Cindro \\ Jožef Stefan Institute, Ljubljana, Slovenia
}

S.H. Abidi, A.A. Affolder, M. Arratia, V. Cindro, B. Ciungu, K. Dette, Z. Doležal, C. Escobar, V. Fadeyev, C. García, I.M. Gregor, K. Hara, L.B.A. Holmes, Y. Ikegai, P. Kodys, G. Kramberger, J. Kroll,C. Lacasta, V. Latonova, D. Madaffari, I. Mandić, M. Mikestikova, M. Mikuž, M. Miñano, K. Nakamura, R.S. Orr, D. Rodriguez, E. Rossi, U. Soldevilla, J. Suzuki, R. Teuscher, Y. Unno, S. Wada

On behalf of ITk collaboration 


\section{Outline}

- Motivation

- Irradiations

- C/V and I/V measurements

- CCE measurements with Alibava setup

- Edge TCT measurements

- Conclusions 


\section{Fluences in ATLAS ITk:}

- Strips: 4 barrel layers and 6 forward disks $\left(165 \mathrm{~m}^{2}\right) \quad 40 \mathrm{~cm} \leq r \leq 100 \mathrm{~cm}$

- Strip sensors with $75.5 \mu \mathrm{m}$ pitch in barrel and ranging from $69 \mu \mathrm{m}$ to $85 \mu \mathrm{m}$ in disks

- Peak instantaneous luminosity up to $L=7.510^{34} \mathrm{~cm}^{-2} \mathrm{~s}^{-1}$ (pile-up $\approx 200$ )

- The predictions (from ATLAS TDR) of particle fluences and ionising doses for the ITk layout assume an integrated luminosity of $3000 \mathrm{fb}^{-1}$ :

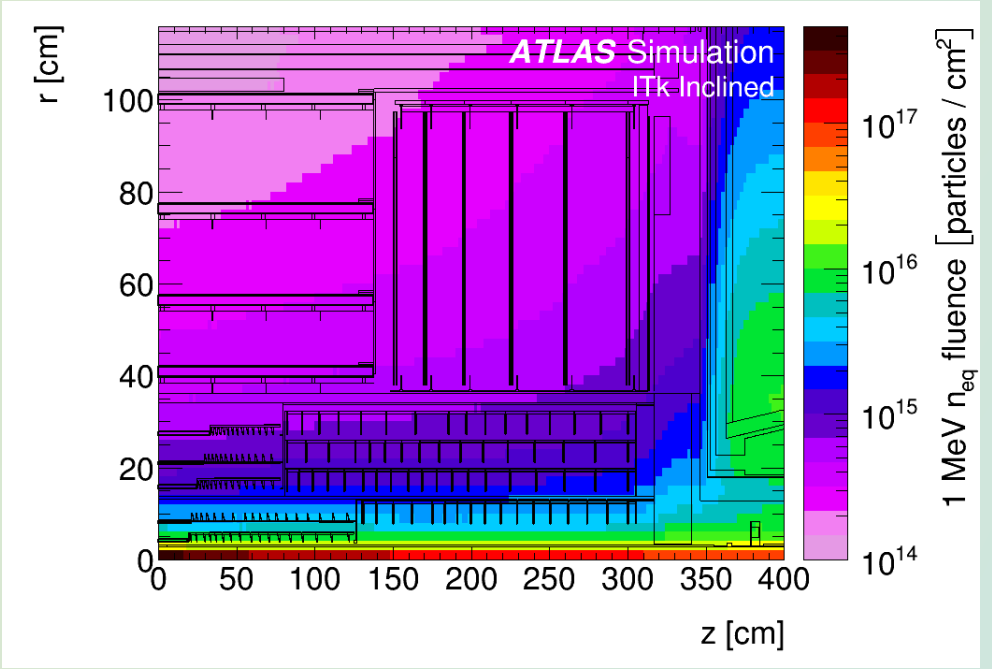

\begin{tabular}{|c|c|c|c|c|c|}
\hline Location & $\mathbf{1 ~ M e V ~}$ n. eq. $\left(\mathbf{c m}^{-2}\right)$ & Protons & Pions & Neutrons & Dose (kGy) \\
\hline \hline$(\mathrm{r}=28 \mathrm{~cm} ; \mathrm{z}=0 \mathrm{~cm})$ & $6.9 \mathrm{e}+14$ & $7.8 \%$ & $38.5 \%$ & $53.7 \%$ & 329 \\
\hline \hline$(\mathrm{r}=28 \mathrm{~cm} ; \mathrm{z}=117 \mathrm{~cm})$ & $8.9 \mathrm{e}+14$ & $13 \%$ & $31.8 \%$ & $55.2 \%$ & 418 \\
\hline \hline LS $(\mathrm{r}=100 \mathrm{~cm} ; \mathrm{z}=0 \mathrm{~cm})$ & $1.7 \mathrm{e}+14$ & $6 \%$ & $13 \%$ & $81 \%$ & 34 \\
\hline \hline LS $(\mathrm{r}=100 \mathrm{~cm} ; \mathrm{z}=117 \mathrm{~cm})$ & $2.1 \mathrm{e}+14$ & $6.2 \%$ & $10 \%$ & $83.8 \%$ & 38 \\
\hline \hline
\end{tabular}

The maximum $1 \mathrm{MeV}$ neutron equivalent fluences for the barrel and strip end-cap detectors are predicted to be $2.810^{14} \mathrm{~cm}^{2}$ and $8.210^{14} \mathrm{~cm}^{2}$, respectively. In addition a safety factor 1.5 is required!

Strips at $r>40 \mathrm{~cm} \rightarrow$ neutrons have the largest contribution to the NIEL! 


\section{Miniature sensors:}

The miniature sensors with $\mathrm{n}$-strips in $\mathrm{p}$-bulk:

- miniature sensors produced by Hammamatsu on the same high resistivity FZ 6 inch wafers as prototypes of full size RO (the first ring in ATLAS strip endcap) sensors (previous presentation)

- outer dimension of $10 \mathrm{~mm}^{2} \times 10 \mathrm{~mm}^{2}, \approx 325 \mu \mathrm{m}$ thick

- 104 readout strips, $8 \mathrm{~mm}$ long

- three different pitches to match the variation of pitches on "Stereo Annulus" sensors

- $75.5 \mu \mathrm{m}$ (default)

- $70 \mu \mathrm{m}$ (narrow)

- $84 \mu \mathrm{m}$ (wide)

- $\quad A C$ and DC coupled

- distributed to different labs for irradiations, electrical tests and charge collection measurements

- irradiations with protons and distribution to labs

- CV/IV measurements / Prague

- proton irradiated CCE measurements in Japan and Valencia

- neutron irradiated CCE and TCT measurements in Ljubljana

- distribution of work:

\begin{tabular}{|l|l|l|}
\hline p irrad & CYRIC (Japan) & \\
\hline$n$ irrad & Ljubljana & \\
\hline C/ I I/V & Prague & Japan \\
\hline CCE $p$ & Japan & Valencia \\
\hline CCE $n$, Edge TCT & Ljubljana & \\
\hline
\end{tabular}

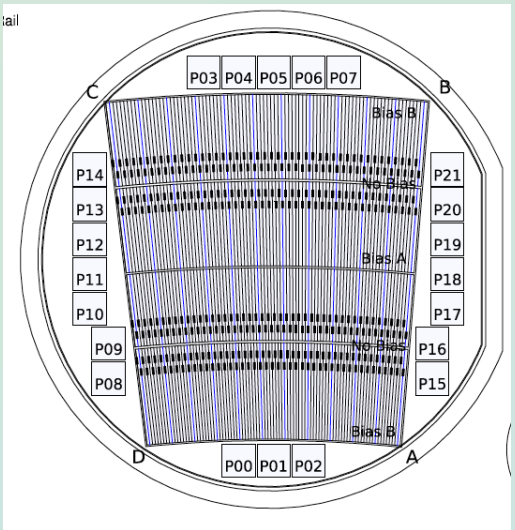




\section{Irradiations with $70 \mathrm{MeV}$ protons at CYRIC (Japan):}

- temperature $\approx-15^{\circ} \mathrm{C}$

- scanning with sample movement

- irradiations $\approx 1 \mathrm{hr}$

- dosimetry using $\mathrm{Al}^{27}(\mathrm{p}, 3 \mathrm{pn}) \mathrm{Na}^{24}$ spallation reaction

- damage factor from RD50 (1.47)

- equivalent fluence uncertainty $10 \%$

- 3 fluences: $5.6,11$ and $22 \times 10^{14} \mathrm{n}_{\mathrm{eq}} \mathrm{cm}^{-2}$

- storage in the fridge after irradiation

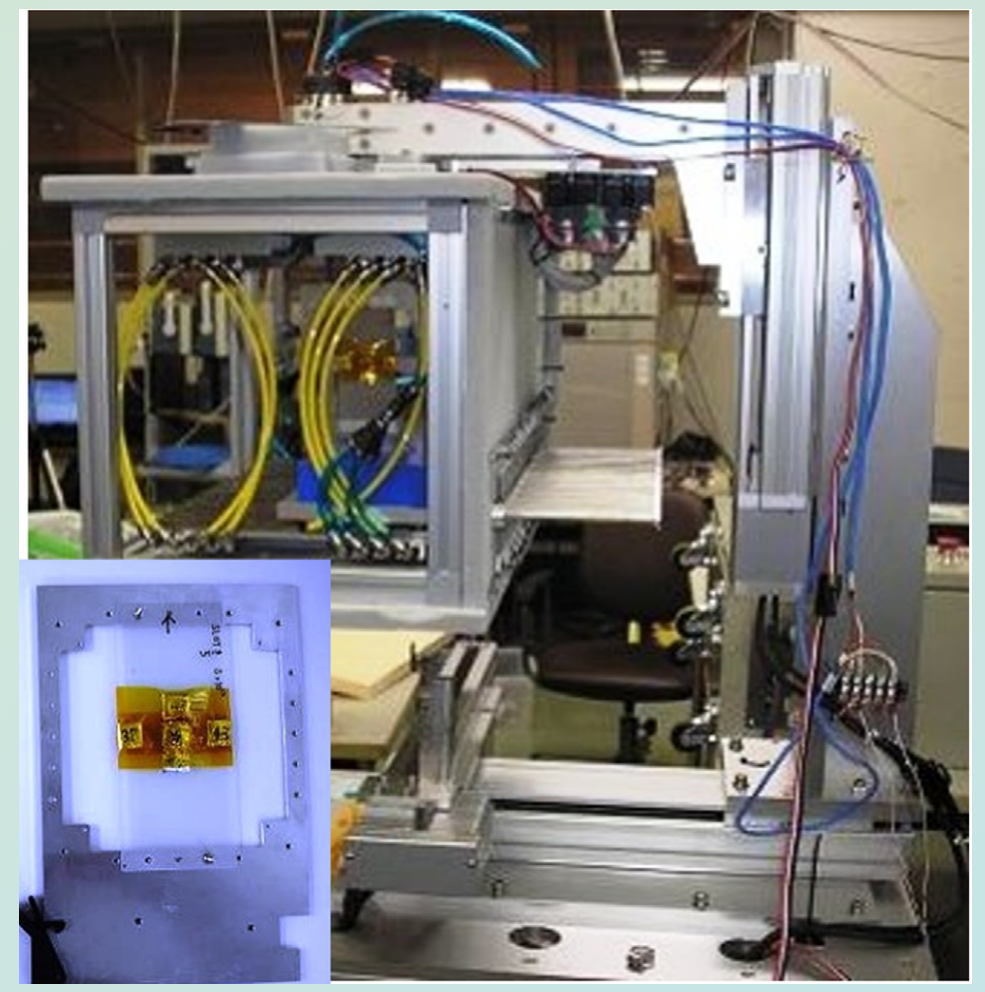




\section{Irradiations with neutrons:}

- TRIGA reactor at Jožef Stefan Institute, Ljubljana

- damage factor $\approx 0.90$ for fast neutrons

- irradiation flux $\approx 1.610^{12} \mathrm{n}_{\mathrm{eq}} \mathrm{cm}^{-2} \mathrm{~s}^{-1}$ at full power $(250 \mathrm{~kW})$

- uncertainty of fluences $\pm 10 \%$

- equivalent fluences: $5 \times 10^{14}, 10^{15}$ and $2 \times$ $10^{15} \mathrm{n}_{\text {eq }} \mathrm{cm}^{2}$

- Approx. 2 hours at RT before $1^{\text {st }}$ CCE measurement
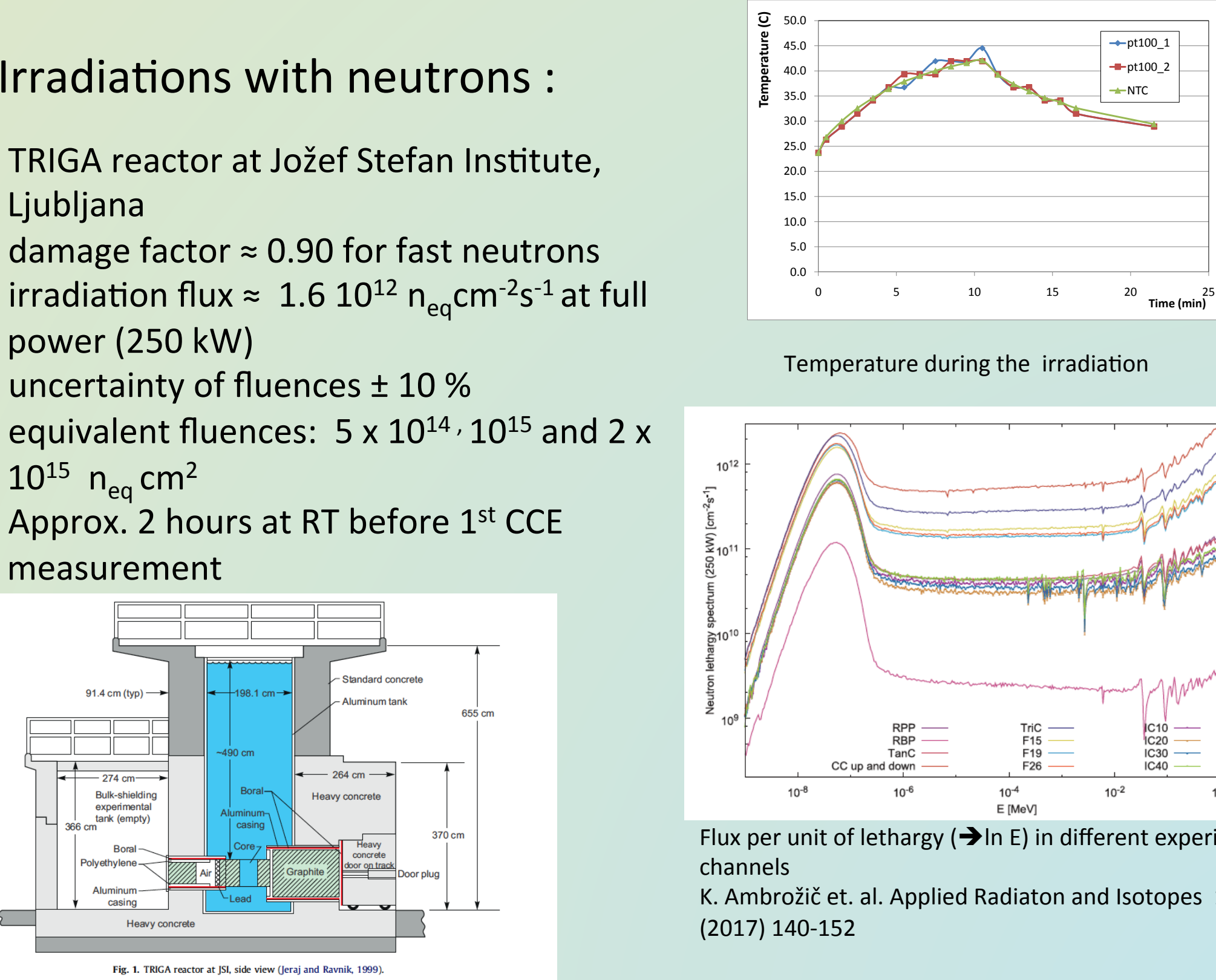

Temperature during the irradiation

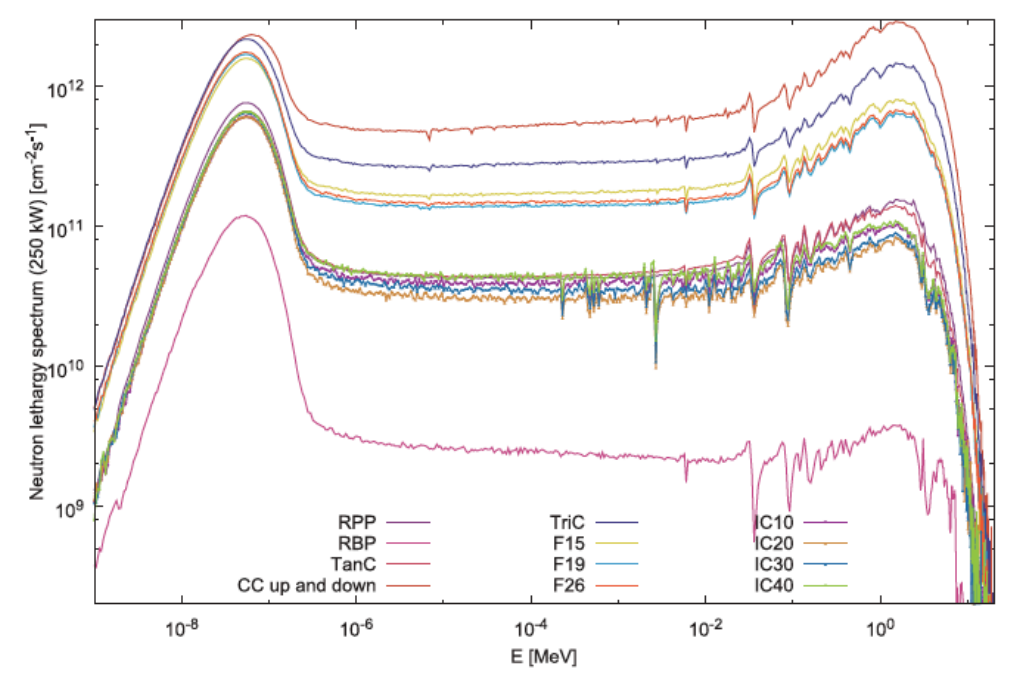

Flux per unit of lethargy $(\rightarrow \ln E)$ in different experimental channels

K. Ambrožič et. al. Applied Radiaton and Isotopes 130 (2017) 140-152 


\section{Total Leakage Current: Pre- and post proton irradiation ATLAS12EC}

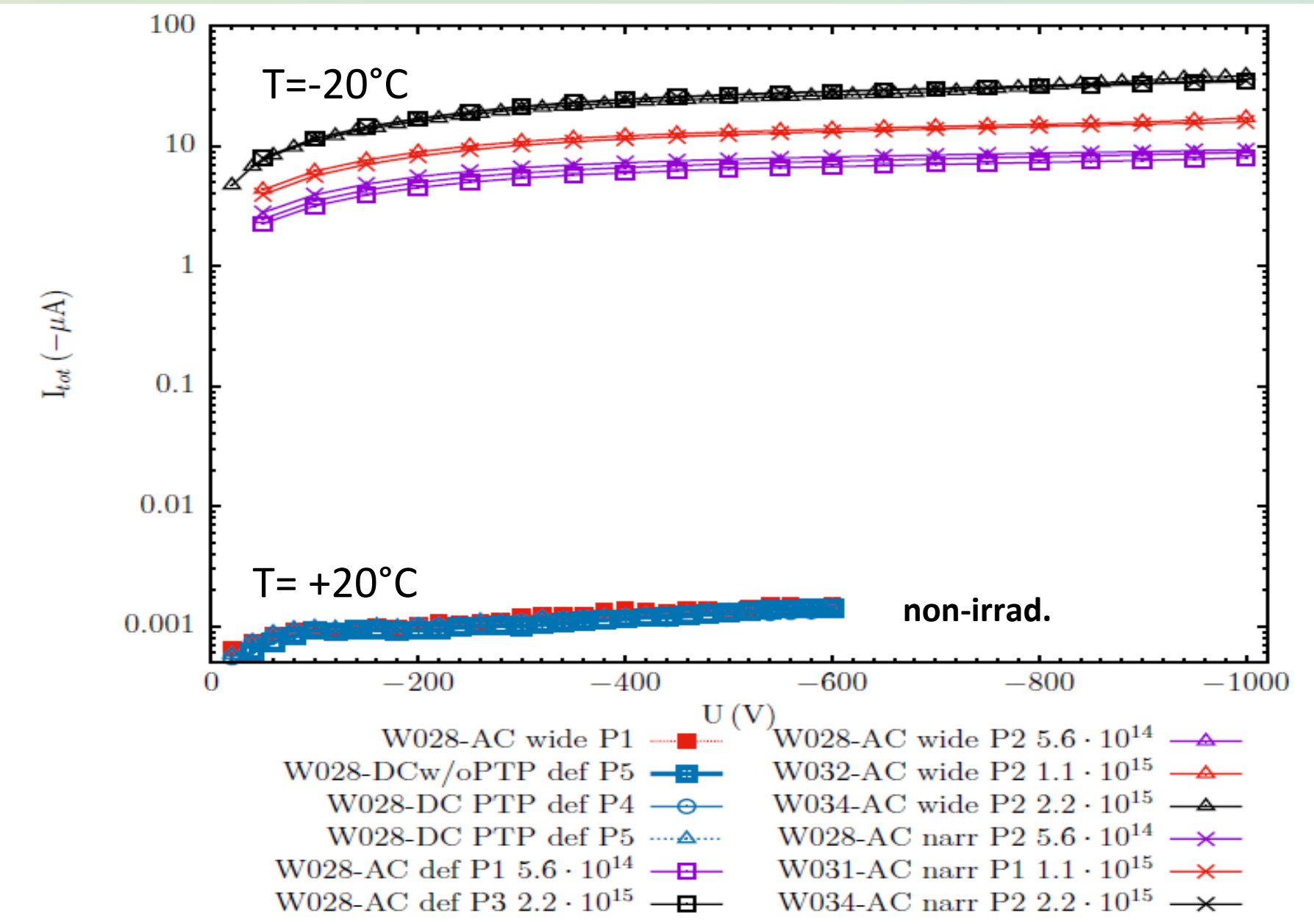

- Smooth behaviour up to $600 \mathrm{~V} / 1000 \mathrm{~V}$

- No breakdown voltage observed in measured interval

- Leakage current increases 


\section{Leakage current per $\mathrm{cm}^{2}$ of proton irradiated mini's Comparison A12A, A12M, A07 and A12EC}

- Total number of sensor tested 126:

- Proton Irradiations: Karlsruhe, Birmingham, CYRIC

- Temperature normalized to $-15^{\circ} \mathrm{C}$,

$$
I\left(T_{-15}\right)=I\left(T_{M}\right) *\left(T_{-15} / T_{M}\right) 2 * \exp \left[-E / 2 k_{B}^{*}\left(1 / T_{-15}-1 / T_{M}\right)\right] \text {, with } E=1.2 e V
$$

- Sensors annealed 80 minutes at $60^{\circ} \mathrm{C}$

- Current normalized to $1 \mathrm{~cm}^{2}$ area;

- Active area - Inner dimension of bias ring $\Rightarrow \mathrm{A}(\mathrm{EC})=0.69 \mathrm{~cm}^{2}, \mathrm{~A}($ barrel, $\mathrm{A} 12 \mathrm{EC})=0.637 \mathrm{~cm}^{2}$

- Alfa $\left(+20^{\circ} \mathrm{C}\right)=4 \mathrm{e}-17 \mathrm{~A} / \mathrm{cm}$, temperature correction coefficient between $+20^{\circ} \mathrm{C}$ and $-15^{\circ} \mathrm{C}: 33$

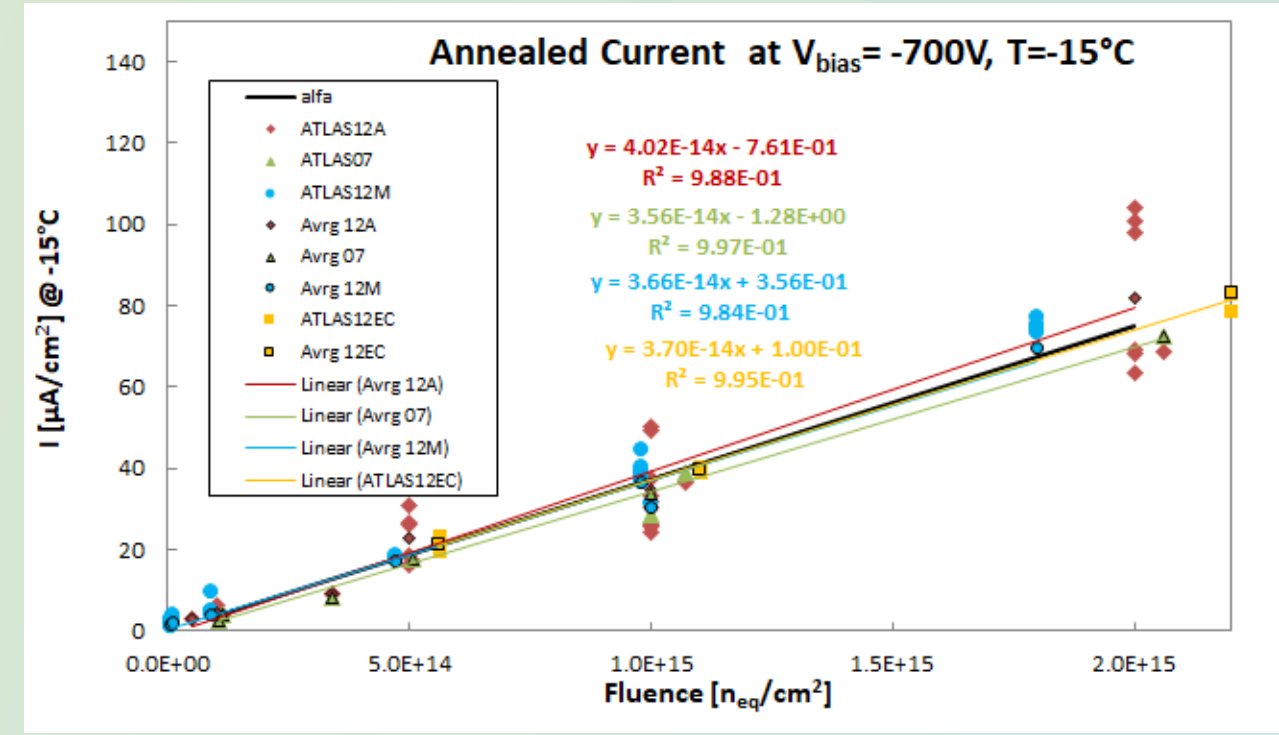

- Leakage currents of new A12EC (R0) sensors are consistent with A12A, A12M and A07 


\section{Characteristics for Full Depletion Voltage (FDV) estimation: Pre- and post proton irradiation ATLAS12EC - RO}

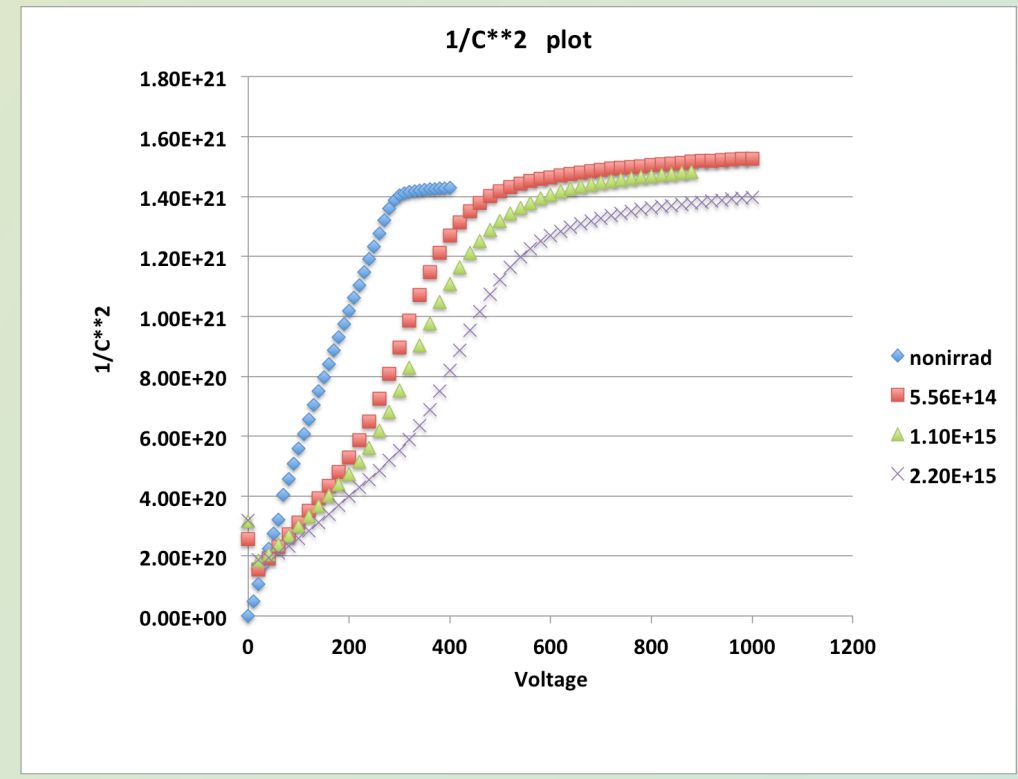

\section{Non-irradiated sensors:}

- FDV = $280-290 \mathrm{~V}(\rho=3.1 \mathrm{k} \Omega \mathrm{cm})$

- Cbulk at full depletion is $25 \mathrm{pF}$

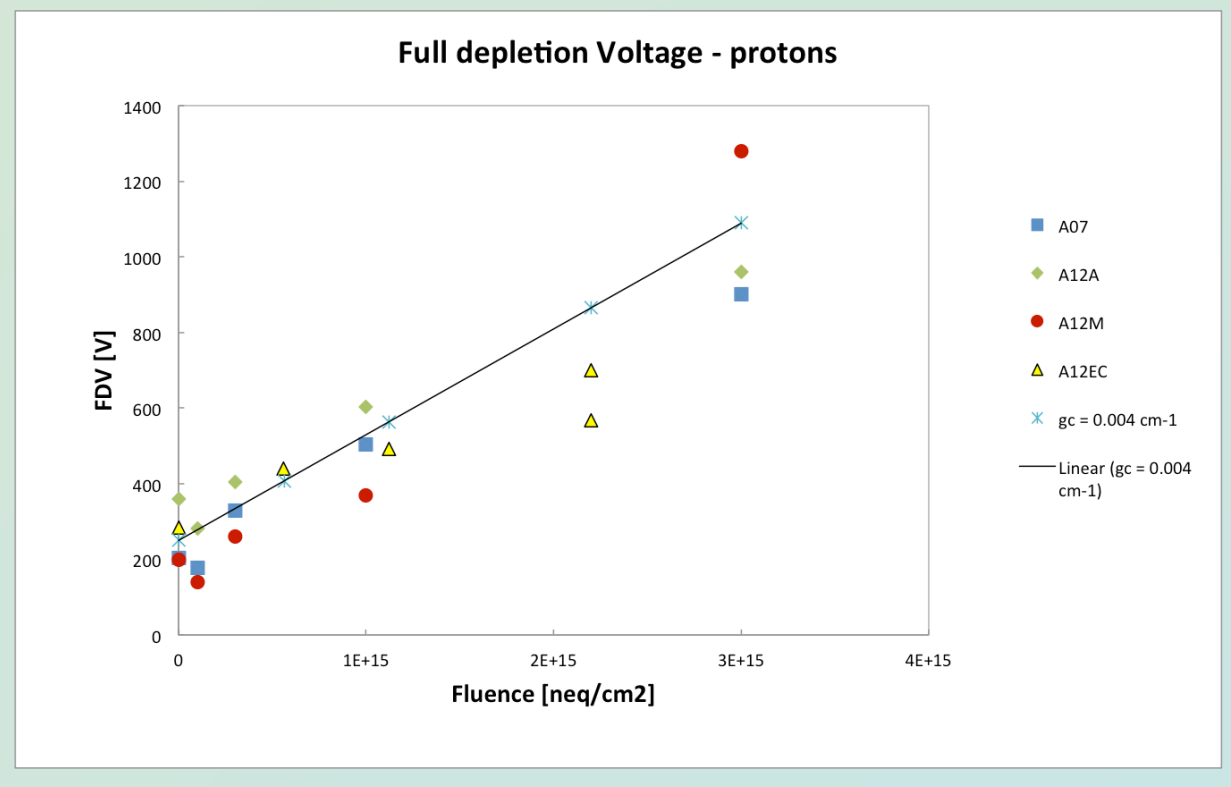

Irradiated sensors:

- "FDV" increased up to $600 \mathrm{~V}$ at $2.2 \mathrm{e} 15 \mathrm{n}_{\mathrm{eq}} / \mathrm{cm}^{2}$

- increase comparable to other batches 


\section{CCE measurements (Alibava setup)}

- based on analogue Beetle chip

- 25 ns peaking time

- $40 \mathrm{MHz}$ clock

- ${ }^{90} \mathrm{Sr}$ source

- different cooling systems (cold box, Peltier)

- triggering with scintillator

- same settings of Alibava in all labs (kazu.ini)

- normalization to MIP MPV - 23100 e-h pairs in $300 \mu \mathrm{m}$ of depleted unirradiated silicon (77 pairs/ $\mu \mathrm{m})$

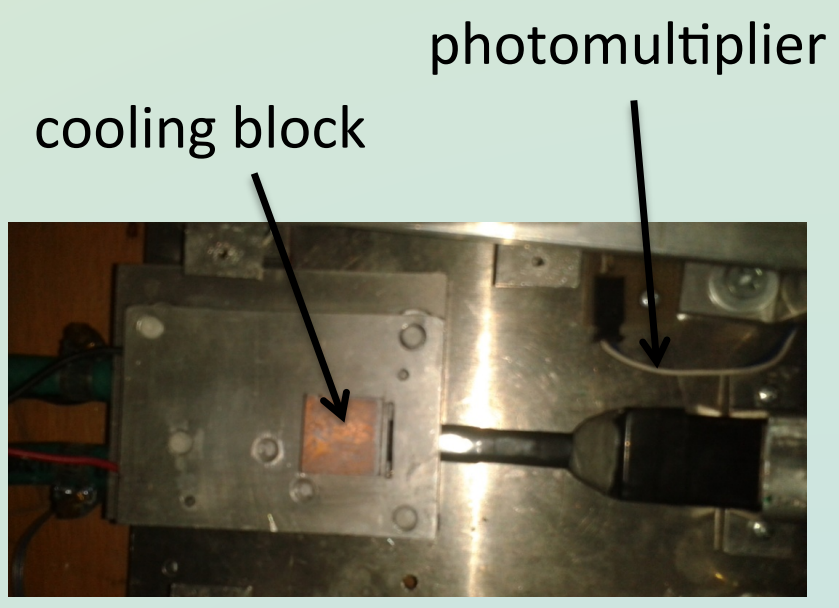

${ }^{90} \mathrm{Sr}$ source

holder
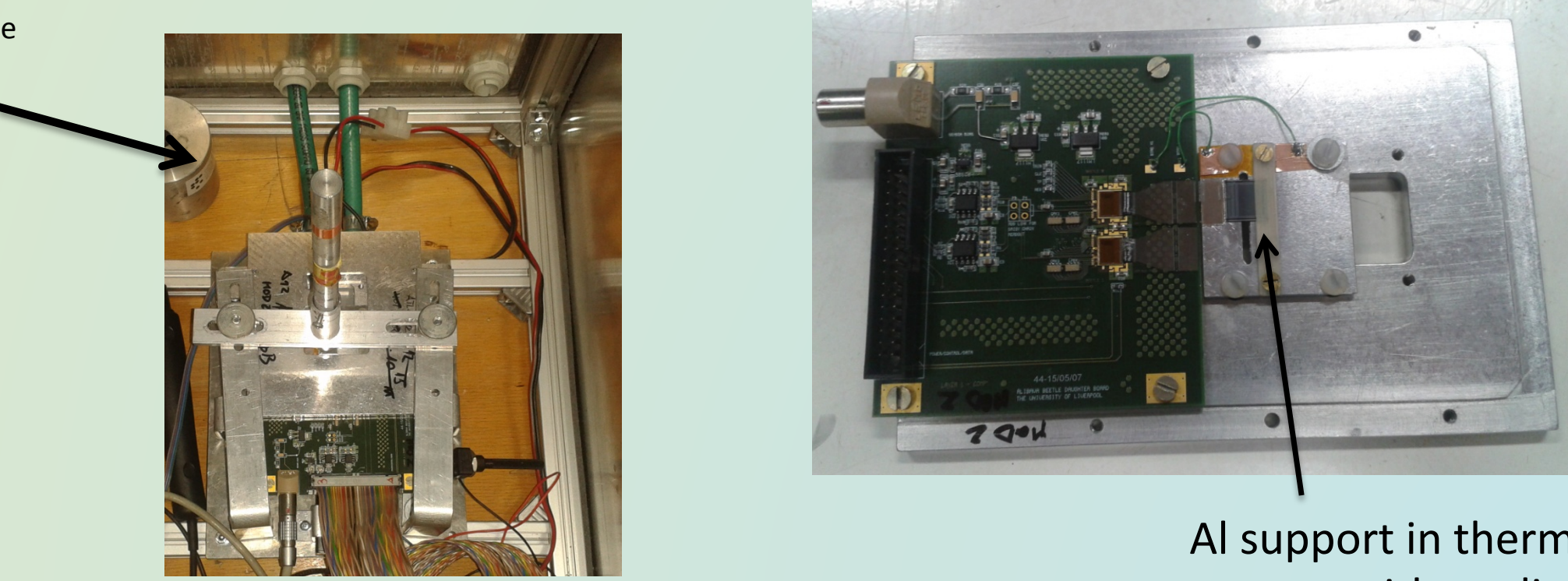

Al support in thermal 


\section{Neutron irradiated sensors - before (dashed) and after (solid) annealing $80 \mathrm{~min}$ at $60^{\circ} \mathrm{C}$ :}
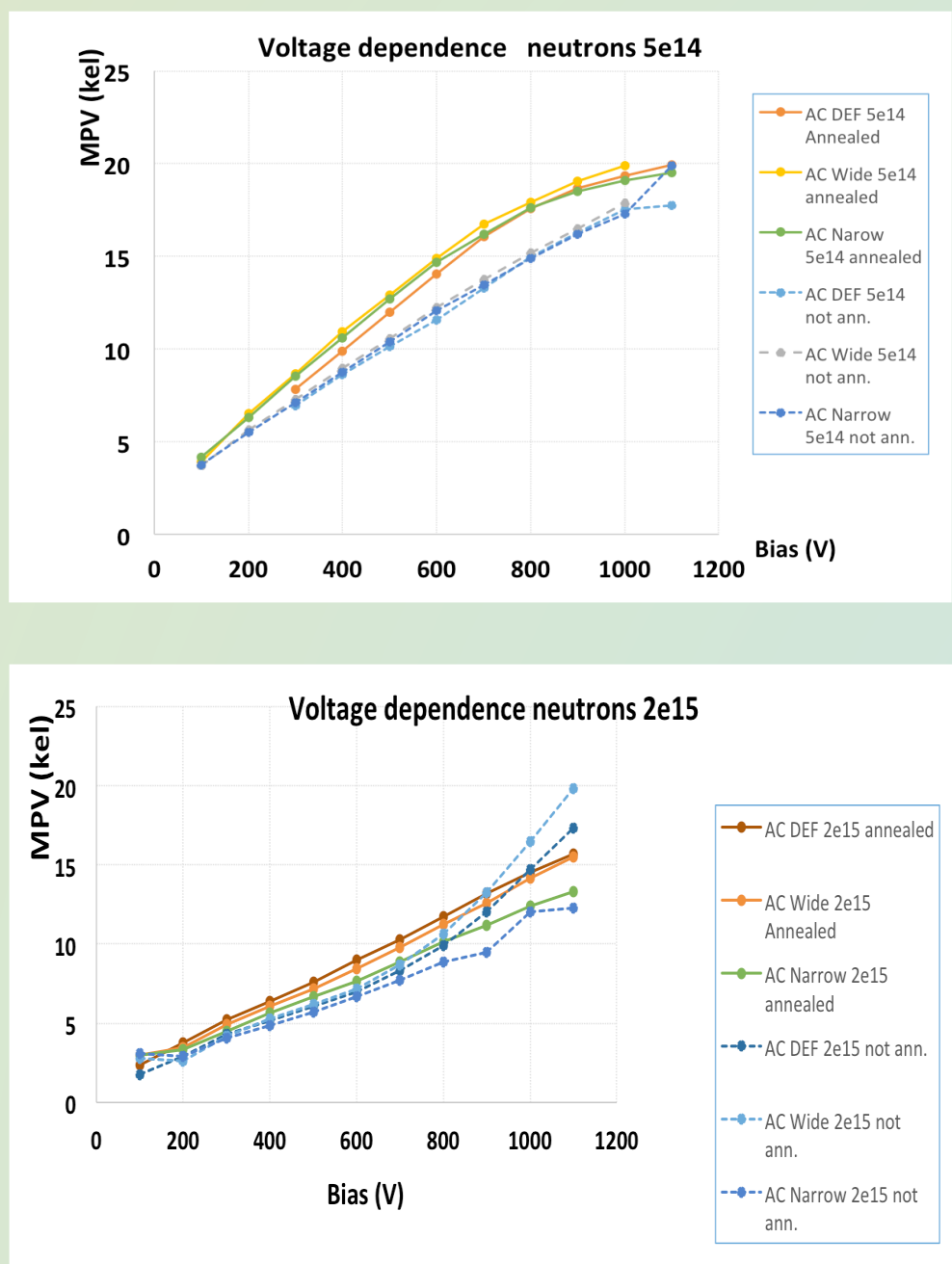

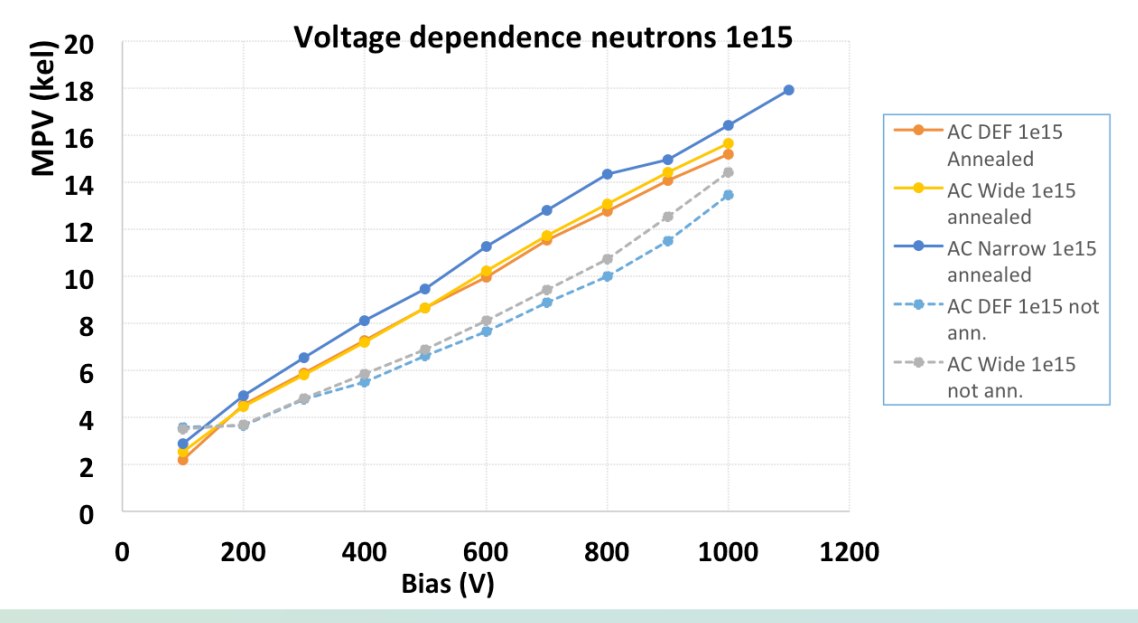

- annealing increases charge

- no difference for different pitches and voltages below $800 \mathrm{~V}$

- wider pitch gives higher signal before annealing at the highest fluence and above 800V indication of multiplication

- this effect disappears after annealing (decrease of space charge) 


\section{Long term annealing at $60^{\circ} \mathrm{C}$ :}

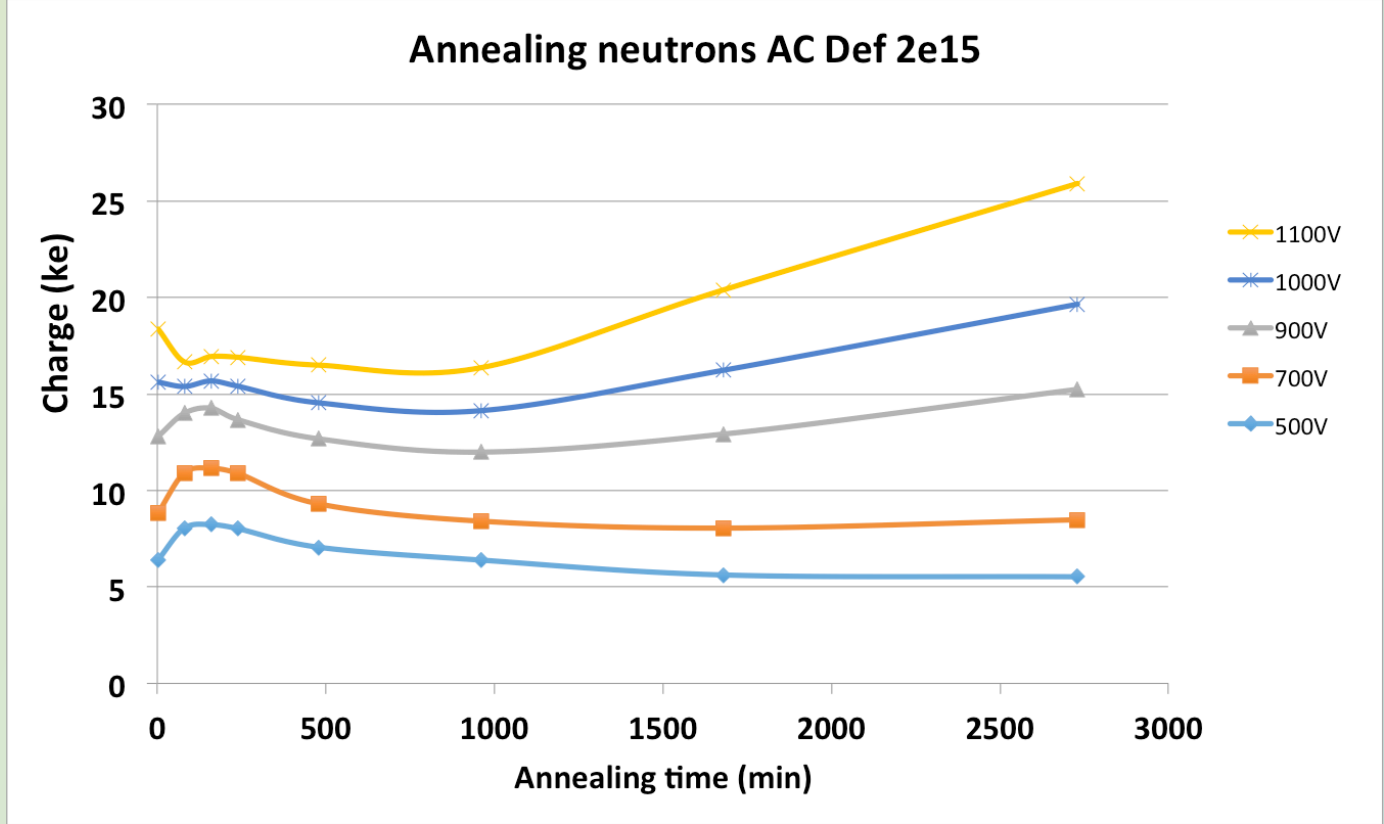

- short term - decrease of space charge and trapping improves CCE except at voltages above $1000 \mathrm{~V}$ where multiplication disappears with annealing

- long term - multiplication at higher voltages increases charge 


\section{Fluence dependence neutrons:}
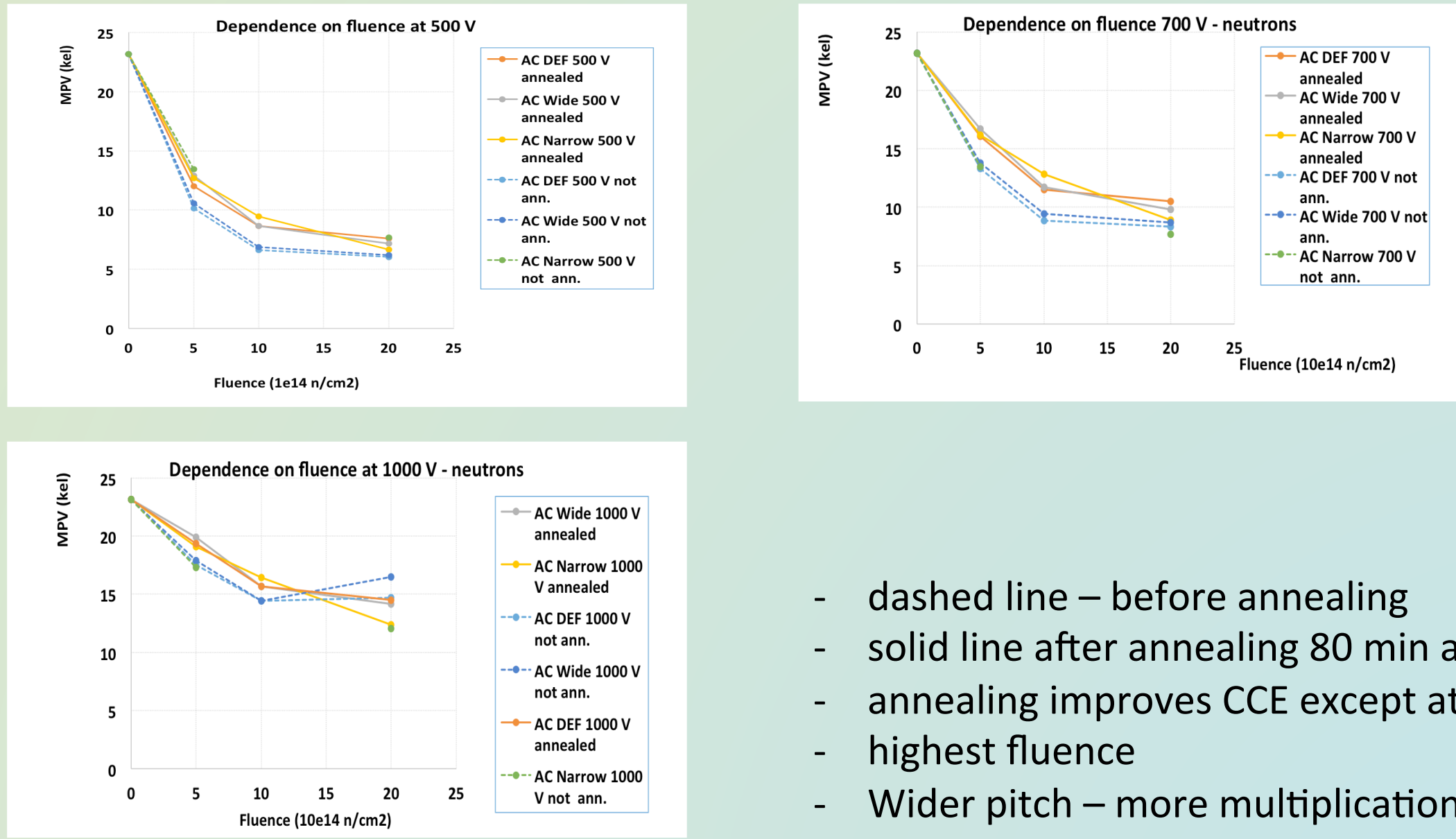

- dashed line - before annealing

- solid line after annealing $80 \mathrm{~min}$ at $60^{\circ} \mathrm{C}$

- annealing improves CCE except at the

- highest fluence

- Wider pitch - more multiplication! 


\section{Comparison with A12:}

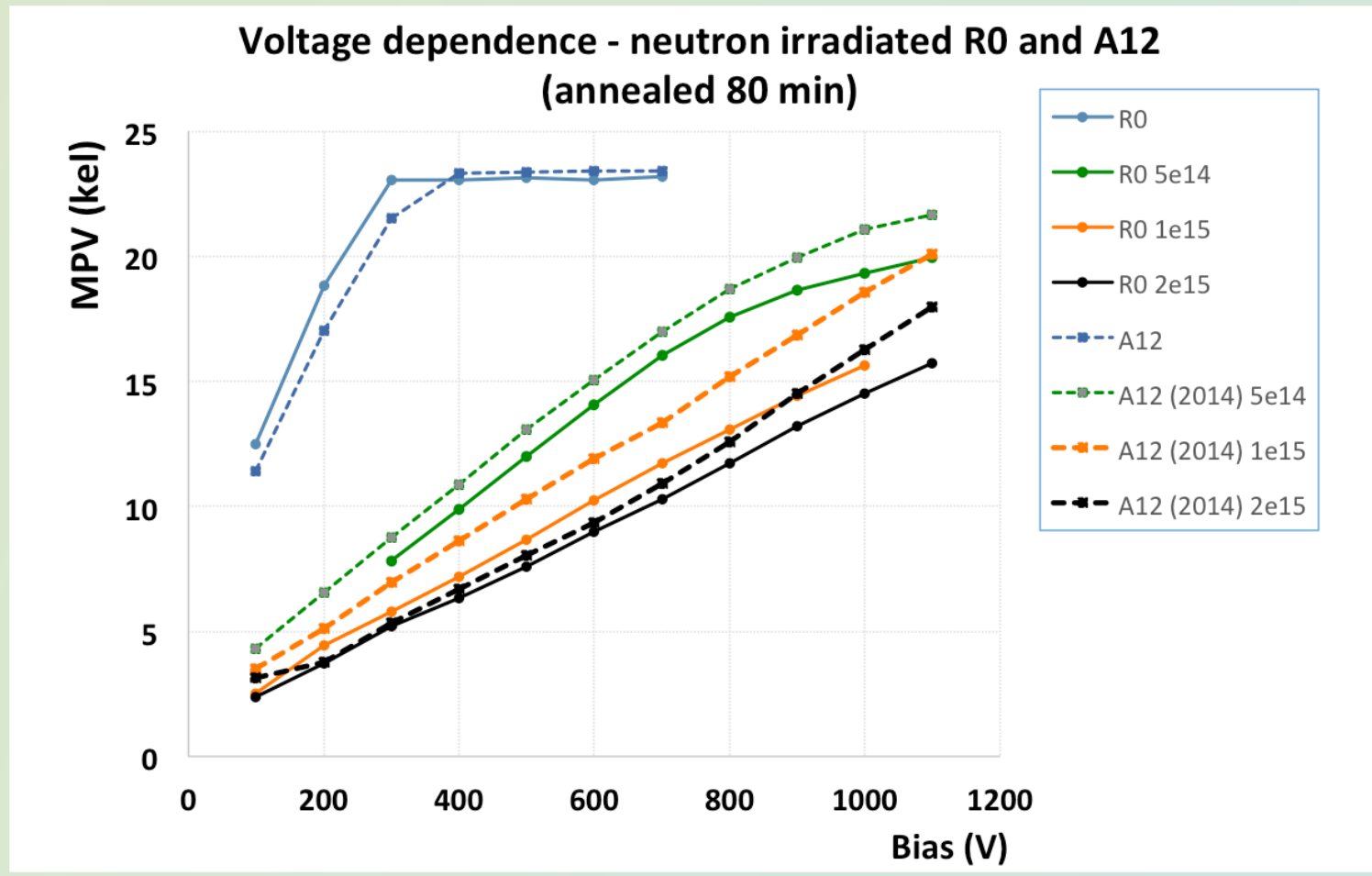

RO AC def sensors full lines A12 dashed lines

- A12 (measured in 2014) - lower nominal resistivity $(3.1 \mathrm{k} \Omega$ ) than R0 (3.5 k $\Omega)$ 


\section{Proton irradiated sensors:}

- not annealed samples - dashed lines

- annealed samples - full lines
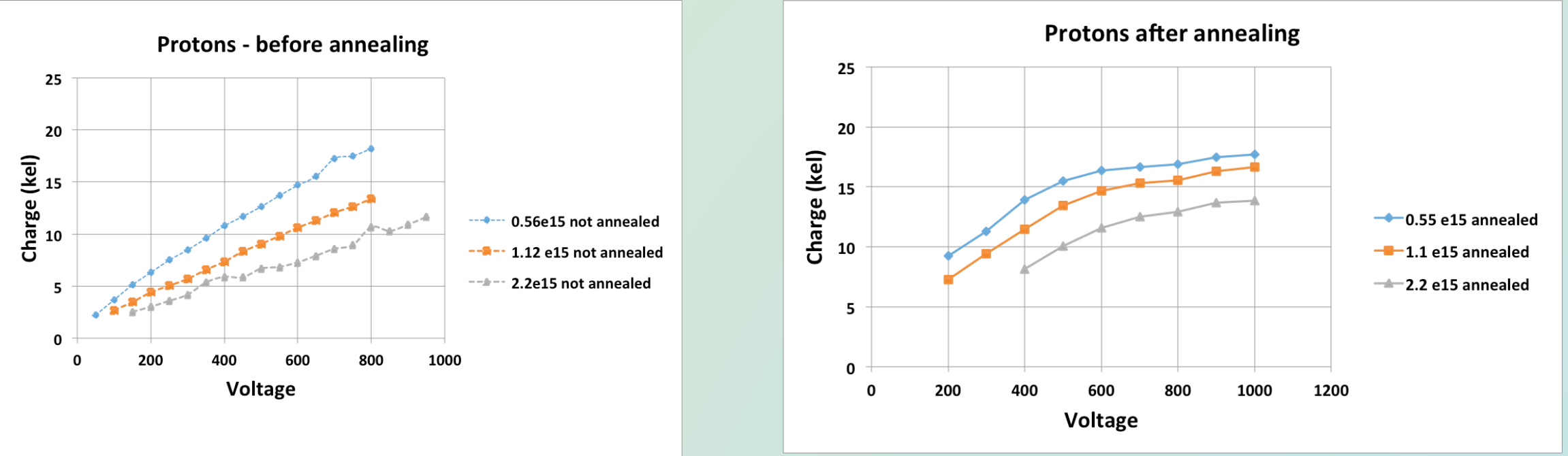

- 80 min annealing at $60^{\circ} \mathrm{C}$ increases collected charge 


\section{Fluence dependence}
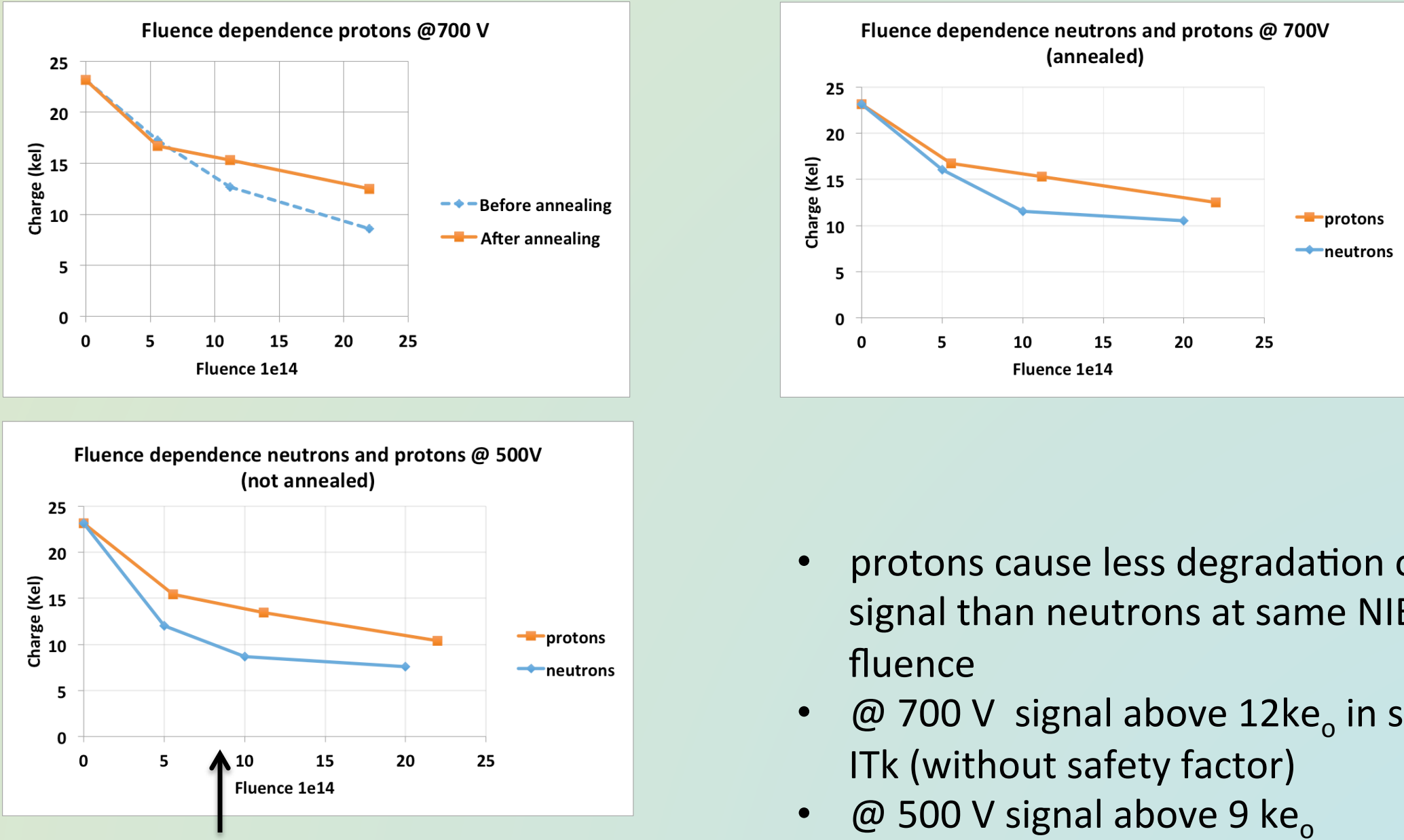

- protons cause less degradation of signal than neutrons at same NIEL fluence

- @ 700 V signal above $12 \mathrm{ke}_{\mathrm{o}}$ in strip ITk (without safety factor)

- @ 500 V signal above 9 ke。

ITk largest fluence 


\section{Edge TCT measurements:}
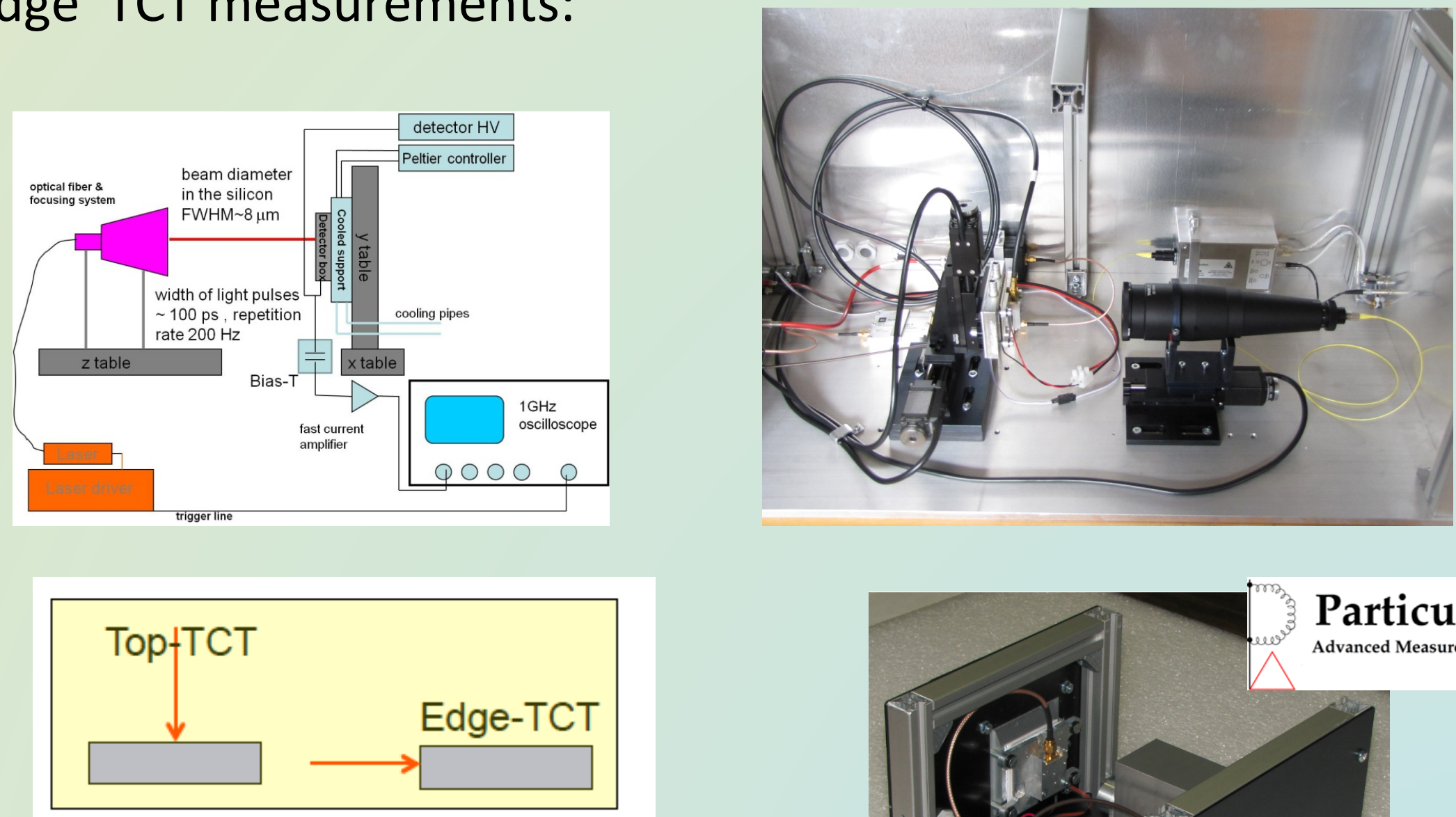

- raise of signal $\rightarrow$ velocity (E field) profile

- integral of signal $\rightarrow$ charge profile

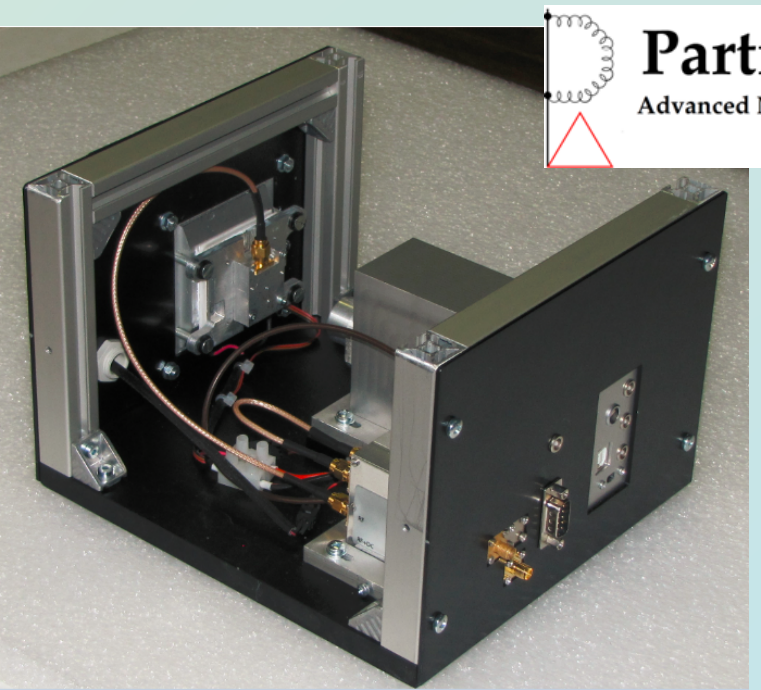




\section{Comparison R0 - ATL12 - before irradiation}

Velocity profile (below depletion:)

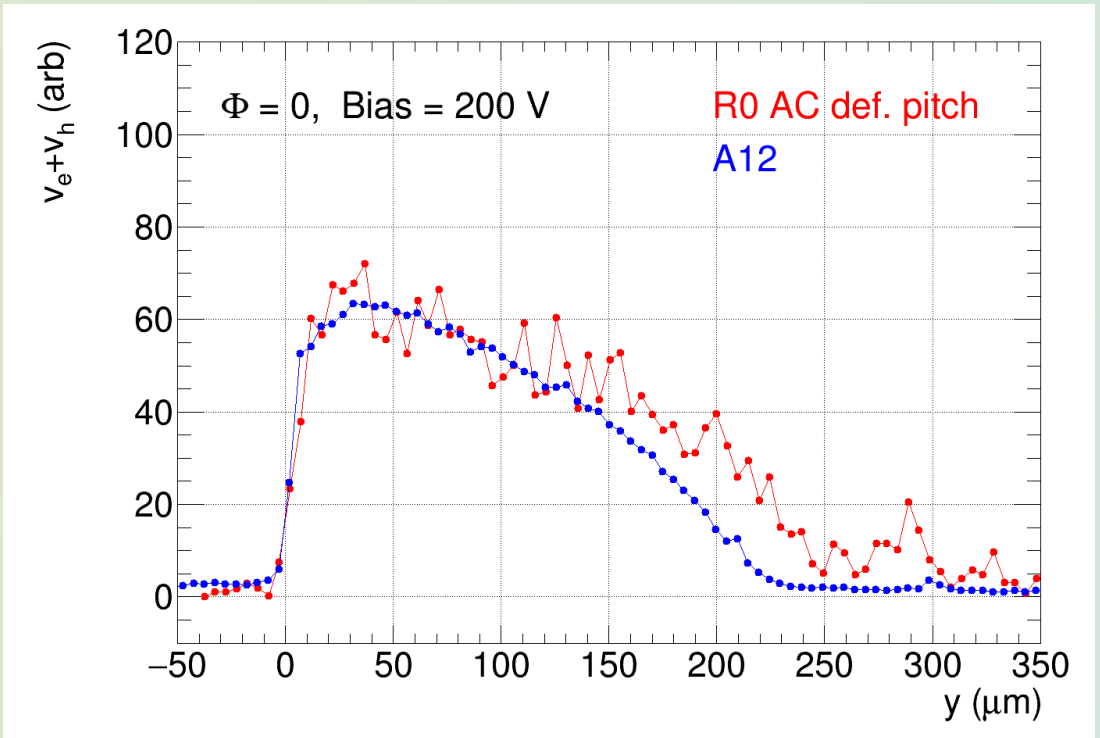

Charge profile (above depletion)

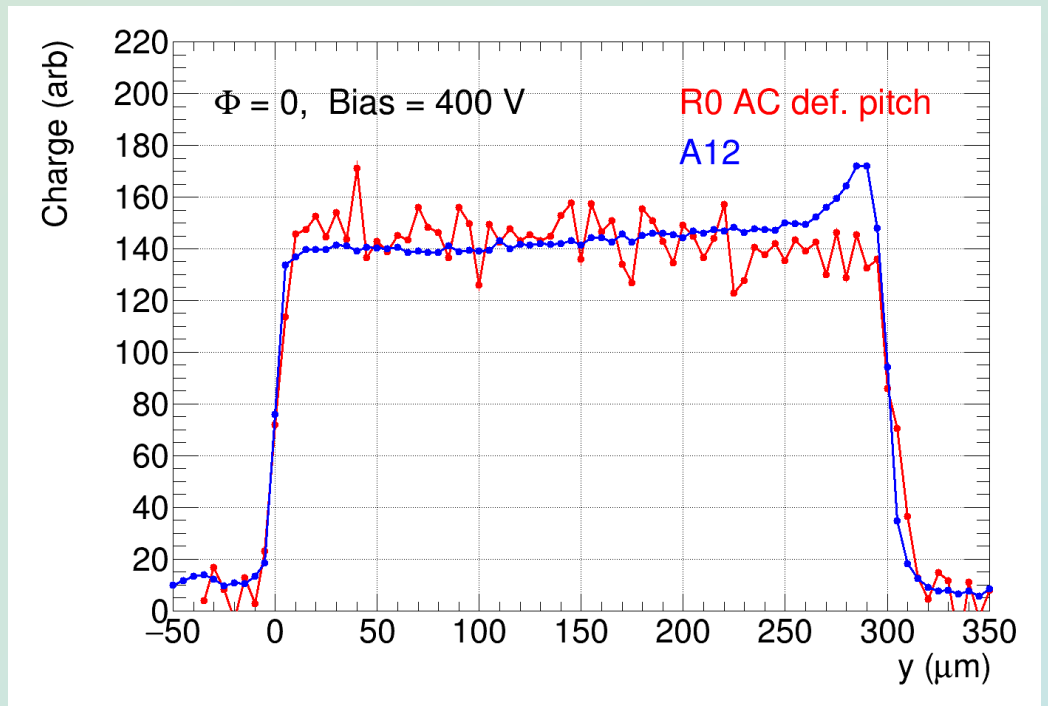

RO sensors were not polished!

Both sensors have same active thickness - $300 \mu \mathrm{m}$

Results consistent with higher resistivity (lower FDV) of R0 material compared to $\mathrm{A} 12$ 


\section{Comparison R0 - ATL12 - after irradiation with neutrons:}

\section{Velocity (E field) profile}

$\Phi=510^{14} \mathrm{ncm}^{-2}$

$\Phi=210^{15} \mathrm{ncm}-2$
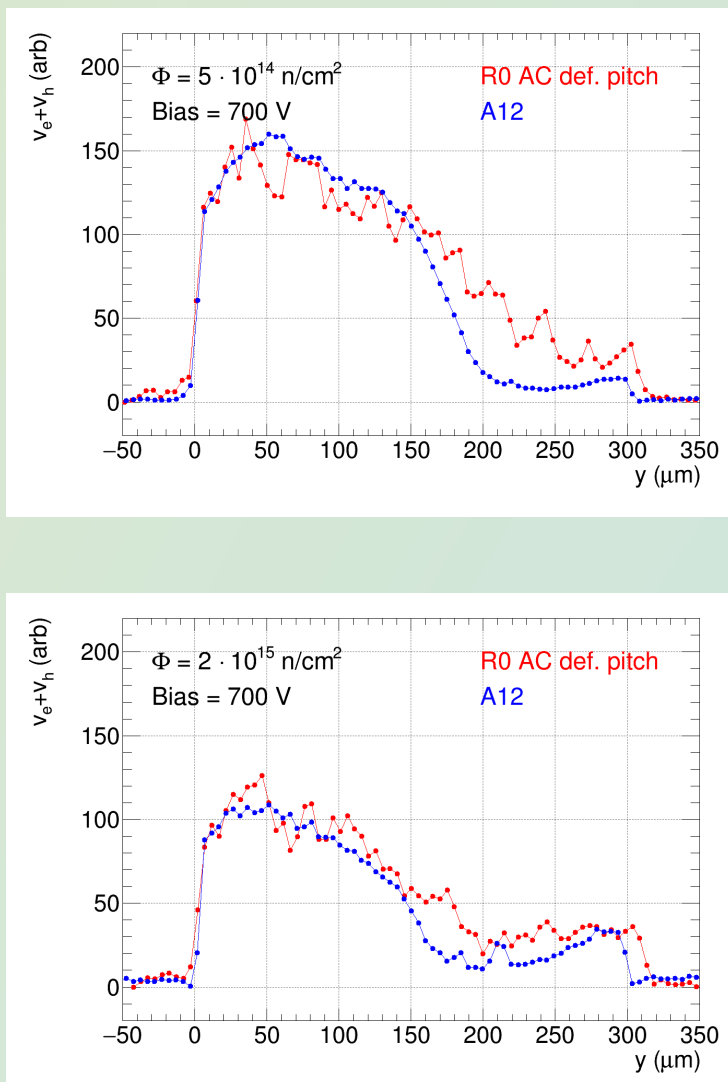

\section{Charge profile}
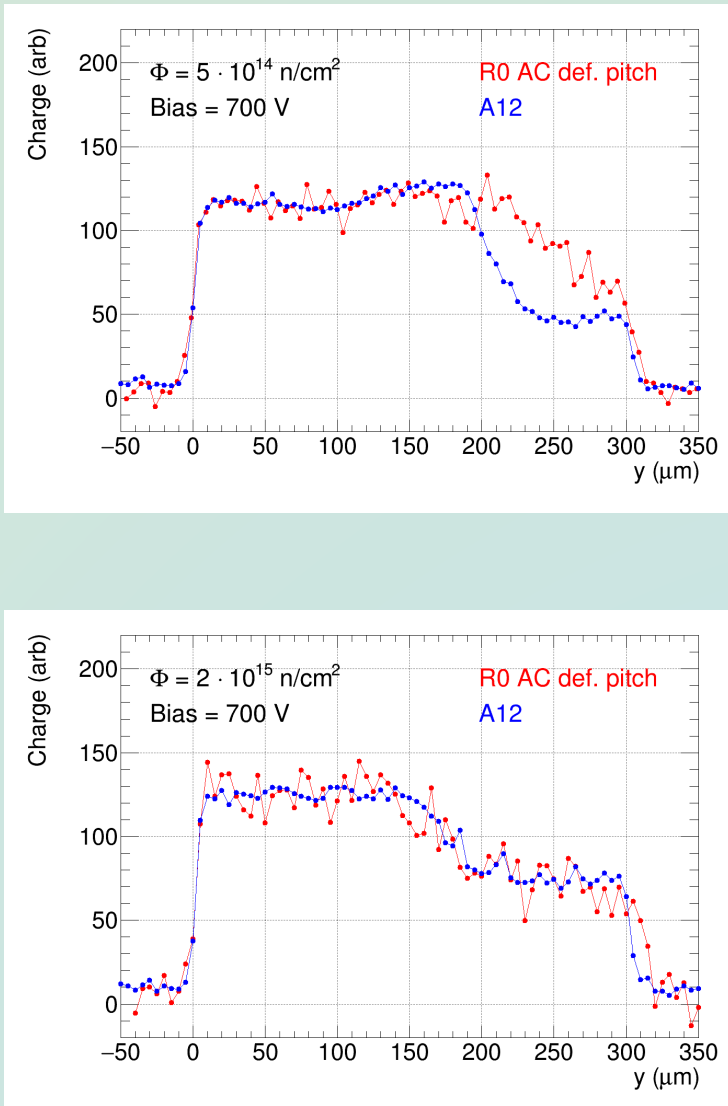

- A12 nominal resistivity $3.1 \mathrm{k} \Omega, \mathrm{R} 0$ nominal resistivity $3.5 \mathrm{k} \Omega$

- $\mathrm{A} 12$ and R0 are more similar at the highest fluence - indication of acceptor removal 


\section{Conclusions:}

- pitch variation does not influence signal up to $800 \mathrm{~V}$ at all fluences

- at the highest fluence multiplication at high voltages

- wider pitch $\rightarrow$ more multiplication

- short term annealing improves signal at lower voltages

- protons cause less degradation of signal than neutrons

- This project has received funding from the European Union's Horizon 2020 Research and Innovation programme under Grant Agreement no. 654168. for irradiations with neutrons at JSI reactor.

- $\quad$ Thanks to team at Cyclotron and Radioisotope Center (CYRIC), Tohoku University for irradiations with protons. 

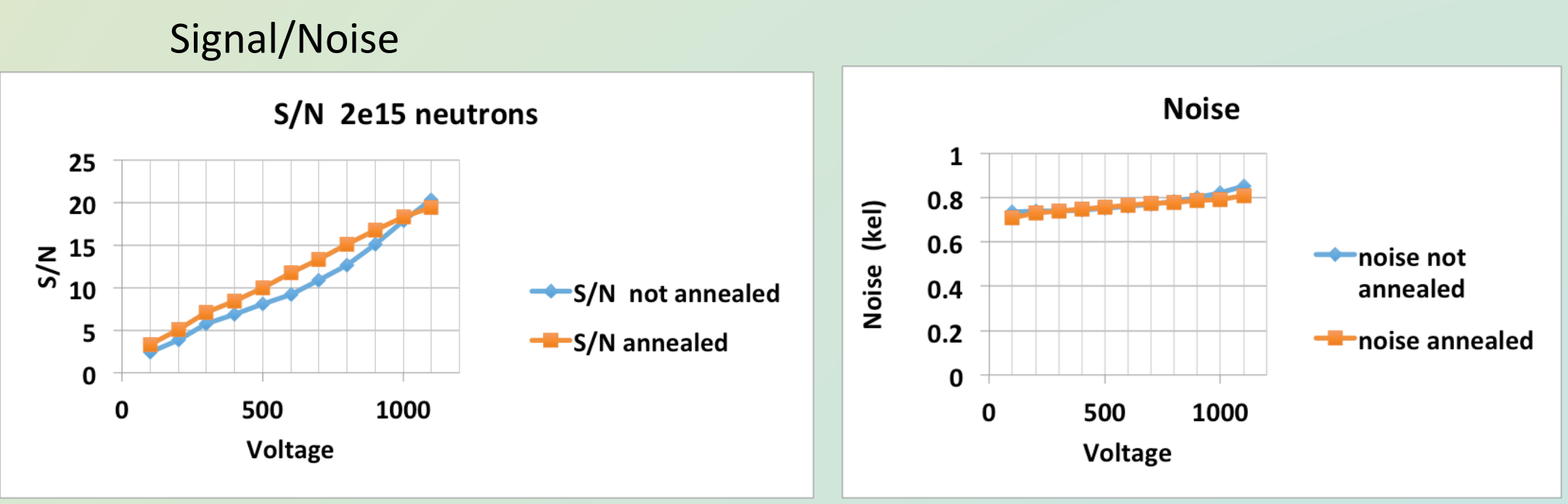

Multiplication increases signal faster than noise

Values are for Beetle chip, short strips, low temperature!! 